\title{
A NEW VERSION OF ELMAN NEURAL NETWORKS FOR DYNAMIC SYSTEMS MODELING AND CONTROL
}

\author{
Dr. Hamdi A. Awad \\ Department of Industrial Electronics and Control Engineering, Faculty \\ of Electronic Engineering, Menouf, 32952, Menoufia University, Egypt. \\ Awadhaa@yahoo.co.uk
}

(Received December 28, 2005 Accepted February 6, 2006)

\begin{abstract}
Elman network is a class of recurrent neural networks used for function approximation. The main problem of this class is that its structure has a set of global sigmoid functions at its hidden layer. That means that if the operating conditions of a process be identified, are changed the function approximation property of the network is degraded. This paper introduces a new version of the Elman network named Elman Recurrent Wavelet Neural Network (ERWNN). It merges the multiresolution property of the wavelets and the learning capabilities of the Elman neural network to inherit the advantages of the two paradigms and to avoid their drawbacks. Stability and convergence property is proven for the proposed network. The paper also develops a model reference control scheme using the proposed ERWNN. The proposed scheme belongs to indirect adaptive control schemes. The dynamic back propagation $(D B P)$ algorithm is employed to train both the two networks structured for the indirect control scheme. This paper derives also the plant sensitivity for adjusting the parameters of the developed controller. The advantages of this new version of ERWNN in modeling and controlling time intensive dynamic processes, are reflected in our simulation results.
\end{abstract}

KEY WORDS: Recurrent neural network, wavelets, respiratory systems.

\section{INTODUCTION}

The nonlinear function mapping properties of neural networks are central to their use in modeling and controlling dynamic systems [1-4]. In general, neural networks can be classified according to their structures into feedforward networks include the multilayer perceptron (MLP) [5], and recurrent networks include the Elman network [6]. They can also be classified according to their learning algorithms include supervised learning [5], unsupervised learning [7] and [8], and reinforcement learning [9]. The Elman recurrent neural network $(\mathrm{ERNN})$ is a type of recurrent networks that has a wide range of applications [10], [11], and [12]. Training the self feedback Elman network with dynamic backpropagation (DBP) algorithm was proposed by Pham and Liu [13]. 
Unlike, the basic Elman network trained by the standard backpropagation (BP) algorithm, the modified Elman trained by DBP was able to model high-order dynamic systems.

Recently, a set of neural networks are structured based on the concepts of the wavelet transform. There are two kinds of Wavelet Neural Networks (WNNs), one with fixed dilation and translation parameters, and the other with adjustable dilation and translation parameters [14] and [15]. The latter realizes the multiresolution property that is very useful for function approximation purposes. The wavelets with coarse resolution can capture the global behavior (low frequency) easily, while the wavelets with fine resolution can capture the local behavior (higher frequency). The performance of a model-based control system depends strongly on the accuracy of the process model used. Many real-time processes have complex, uncertain and non-linear dynamics and so it is difficult to model them mathematically. Because of the function approximation ability of neural networks, much research has been conducted on adapting them for modeling and controlling dynamic systems [4] and [11]. Feedforward neural networks such as MLP and Radial Basis Function (RBF) networks usually does pose a serious problem when it is employed for modeling purposes. This problem can be summarized as follows. If a feedforward network is adopted for the modeling task, then we should know the number of delayed input and output in advance, and feed them as a taped line to the network input. The exact structure of a dynamic system is usually unknown. Besides much taped delayed lines increase the dimension of the input vector that results a large network size. To deal with this problem, interest in using recurrent networks e. g. ERNN for processing dynamic systems has been steadily growing in recent years, [16] and [17].

This paper focuses on the Elman recurrent neural network, ERNN, to overcome the structure identification problem of the feedforward networks mentioned above. The main drawback of using the original ERNN is that it has global sigmoid functions at its hidden layer. That means that if the operating conditions of a process to be modeled are changed, the function approximation property of the Elman network is degraded as mentioned previously. This paper merges the multi-resolution property of the wavelets and the learning capabilities of the Elman network to overcome the limitations of ERNN on modeling and controlling dynamic systems. That results a new version of Elman network named ERWNN that has a universe of discourse covered by a set of local wavelets instead of global sigmoid functions. This is our first motivation. Unlike the traditional Elman recurrent network ERNN [6], the proposed ERWNN has the advantages of better local accuracy, generalization capability and fast convergence as reflected from our simulation results.

This paper also introduces the proposed ERWNN for controlling dynamic systems. It describes a design method for a model reference control structure using the proposed ERWNN. In this structure, two ERWNNs are employed, one is a controller named (ERWNNC) and the other is an identifier called ERWNNI that provides information about the plant be controlled to the former. This scheme is named ERWNN-based indirect control (ERWNN-ICS). The paper derives the plant sensitivity for adjusting the parameters of the controller. The paper also employs the DBP to train both the two networks of the proposed scheme and applies it for controlling time intensive processes. 
Controlling of the oxygen delivery to mechanically ventilated hypoxic patients is a time intensive process that must balance adequate tissue oxygenation against possible toxic effects of oxygen exposure [18] and [19]. Although many researches have been conducted for oxygenation of newborn patients, few researches are carried out for adults. The proposal scheme has been applied to control the oxygen delivery for adults patients. Controlling this process using conventional proportional plus integral (PI) controller needs empirical adjustments for the controller parameters. It means that if the operating conditions of the controlled plant is changed, these parameters should be readjusted. A multi-model adaptive controller (MMAC) [21] and feedforward fuzzy neural network-based indirect control scheme (FNN-ICS) [22], were introduced for controlling this process, however satisfied results have not been achieved. This paper employs this time intensive nonlinear process to test the proposed ERWNN-ICS. Compared with PI, MMAC controllers and FNN-ICS, best results were achieved using the developed ERWNN-ICS.

The motivations of this paper can be concluded as follows:

- Development a new version of ERNN named ERWNN

- Deriving the DBP algorithm to train the proposed ERWNN

- Testing the stability and convergence of the proposed ERWNN

- Development an indirect control scheme based on the proposed ERWNN named ERWNN-ICS

- Deriving the plant sensitivity to adjusting the parameters of the developed ERWNN-ICS

The rest of the paper is organized as follows. Section 2 describes the proposed ERWNN and its training algorithm. Section 3 depicts the modeling simulation results using both the ERNN and the ERWNN. Section 4 details the modeling and controlling of dynamic systems using the proposed indirect control scheme. Training both the two networks of the proposed indirect control scheme is described in section 5. Simulation results on modeling and controlling the respirator dynamic system are depicted in section 6 . Section 7 concludes the paper by summarizing the contributions made.

\section{EIMAN RECURRENT WAVELET NEURAL NETWORK}

This section details wavelet transform. It describes the structure and training algorithm of the introduced ERWNN and derives the DBP algorithm to adapt the parameters of the proposed network.

\subsection{Wavelet Transform}

Unfortunately, most signals encountered in practice, are non-stationary. Such signal requires a time-frequency representation rather just a time or frequency representation. In other words, technologies based on Fourier Transform (FT) or even its modifications still suffer from a set of drawbacks. That is that FTs are not able to analysis or approximate signals with both sharp transitions and slowly varying spectra. Authors who did not realize this fact blindly build their technologies based on FTs. Besides, technologies that depend on FT are quadratic or nonlinear in nature with 
highly computation demand. Wavelet transform is the only linear transform that can analysis or approximate stationary and/or non-stationary signals at varying resolutions.

The basic concepts about wavelet transforms that are relevant to this paper are briefly recalled. The coherent states $\mathrm{g}^{(\mathrm{p}, \mathrm{q})}$ all have the same envelope function $\mathrm{g}$, which is translated by the amount q, and "filled in" with oscillations with frequency p [23]. Wavelets are similar to the $\mathrm{g}^{(\mathrm{p}, \mathrm{q})}$ in that they also constitute a family of functions derived from an single function, and indexed by two labels, one for position, $b$, and the other for frequency, a. That is.

$$
h^{(a, b)}(x)=|a|^{(-1 / 2)} h\left(\frac{x-b}{a}\right)
$$

where $\mathrm{h}$ is a square integral function such that.

$$
c_{h}=\int|y|^{-1}|\hat{h}(y)|^{2} d y<\infty
$$

and $a, b \in R$ and $a \neq 0$. If $\mathrm{h}$ has some decay at infinity, then (2) is equivalent to the requirement $\int h(x) d x=0$. Compared with the coherent state $\mathrm{g}^{(\mathrm{p}, \mathrm{q})}$, wavelets will be a better tool in situations where better time-resolution at higher frequencies, $a<<1$, than at low frequencies, $a>>1$. It is clear that the major different between short-time (windowed) FT and wavelet transform is that, in the latter high frequency components are studied with sharper time resolution than low frequency components.

The link between wavelets and neural networks was introduced by [24]. In general, a set of vectors $\left\{\psi_{j}, j \in J\right\}$ in Hilbert space $H$ for which the sums $\sum_{j \in J}\left|\left\langle\Psi_{j}, f\right\rangle\right|^{2}$ yield upper and lower bounds for the norm $\|f\|^{2}$, is called a frame. Given a frame $\psi_{M, N}$ in the Hilbert space $\mathrm{H}$, for any function $f \in H$, f can be decomposed in terms of the frame elements as follows [15]:

$$
f(x)=\sum_{M, N \in Z^{q}}\left\langle f, S^{-1} \Psi_{M, N}\right\rangle \Psi_{M, N}(x)
$$

where $\mathrm{S}$ is the frame operator $H \rightarrow H$ and $\psi_{M, N}$ is defined below:

$$
\begin{gathered}
\psi_{M, N}(x)=\psi_{a_{1}, b_{1}}^{1}\left(x_{1}\right) \psi_{a_{2}, b_{2}}^{2}\left(x_{2}\right) \ldots \psi_{a_{q}, b_{q}}^{q}\left(x_{q}\right) \\
M^{T}=\left[a_{1}, a_{2}, \ldots, a_{q}\right], N^{T}=\left[b_{1}, b_{2}, \ldots, b_{q}\right], \psi_{a_{i}, b_{i}}^{i}\left(x_{i}\right) \text { can be generated by dilating and }
\end{gathered}
$$

translating the mother wavelet as will be shown in section B. The parameters $a_{i}$ and $b_{i}$ are the dilation and translation respectively.

In most practical applications, the function of interest have finite support, therefore it is possible to truncate infinite number of wavelet frames in (3) to reconstruct the function $\mathrm{f}$ as follows:

$$
f(x) \approx \sum_{M, N}^{Q} w_{M, n} \Psi_{M, N}(x)
$$


where $w_{M, N}=\left\langle f, S^{-1} \Psi_{M, N}\right\rangle$ and $\mathrm{Q}$ is the total number of wavelet function selected. This equivalent to the wavelet neural network introduced by [24]. Initialization and wavelet networks were described in [25] and [26].

\subsection{Structure of ERWNN}

The structure of the proposed ERWNN is similar to the structure of ERNN that is described in [13]. The first motivation of this paper is that it introduces the multiresolution property of the wavelets to the ERNN by replacing the sigmoid function at its hidden layer with a set of wavelet functions defined in (6) as depicted in Fig. 1. The idea of employing wavelets to neural networks described in section A, was borrowed to construct our ERWNN. The novelty of the proposed network can be concluded as follows:

Converting the ERNN from global-support (i.e. sigmoid functions) network, ERNN, to local-support (wavelets) network, ERWNN, results a universal tool for function approximation as will be shown in section 3. This is due to the use of a higher resolution of the space when the data are dense, and a lower resolution when data are sparse.

Merging the multi resolution property of the wavelets to the ERNN, keeps the number of the hidden/context layer approximately six for a complex process. In other words, to obtain similar RMS values using both the two networks, the number of the hidden/context units should be increased using the ERNN compared with the proposed ERWNN.

Referred to Fig. 1, it can be seen that the proposed network, in addition to the input, the hidden units and the output unit, the context units, there are also the link weights. These weights link, respectively, the input / hidden units, the context / hidden units, and the hidden / output units. The function of each unit at a layer in the proposed ERWNN can be described as follows:

Layer-1: The input unit at this layer is only a buffer unit, which passes the signals with out changing them.

Layer-2: Instead of using sigmoid functions [6] at the hidden layer of the traditional ERNN, this paper proposes that each unit in the hidden layer implements the multidimensional wavelets defined in (6):

$$
\varphi_{j}=\prod_{i=1}^{n} h_{i}\left(x_{i}\right)
$$

where $x_{i}=\frac{v_{i}-b_{i}}{a_{i}}, \mathrm{v}_{\mathrm{i}}$ is $\mathrm{i}^{\text {th }}$ input to a hidden unit, $h_{i}($.$) is the \mathrm{i}^{\text {th }}$ daughter wavelet generated by a translation $b_{i}, \quad$ and dilation $a_{i}$ from a mother wavelet $h($.$) . The$ employed mother wavelet is:

$$
h(x)=\left(1-x^{2}\right) \exp \left(-x^{2}\right), \quad a \neq 0
$$

It also proposes the dilation parameter of the wavelets defined in (6) to link the hidden and the context units as shown in Fig. $\mathbf{1}$ and the weights of the traditional network are considered as a scaling parameters. 


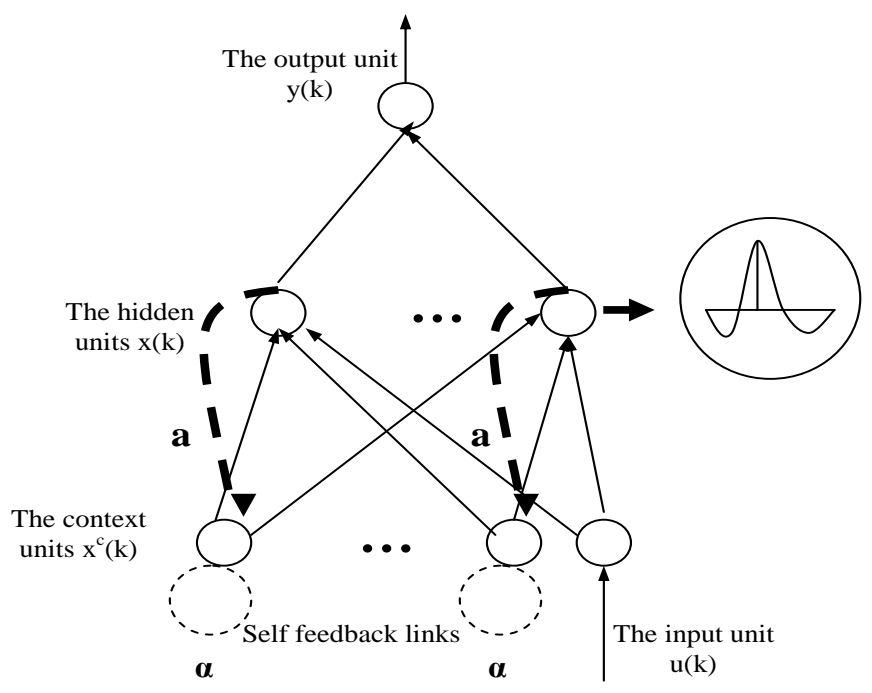

Fig. 1. The modified ERWNN.

Layer-3: This layer represents the final output of the network. At a specific time $k$, the previous activations of the hidden units (at time k-1) and the current input (at time k) are used as inputs to the hidden units. At this stage, the network acts as a feedforward network and propagates theses inputs forward to produce the output similar to the traditional ERNN. This shows that the proposed ERWNN is an approximate realization of ERNN. The function of each unit at a specific layer in the proposed ERWNN is described as follows:

The input of a hidden unit at the hidden layer is:

$$
\begin{aligned}
& v_{i, j}(k)=w_{i, j}^{x}(k-1) x_{j}^{c}(k) \\
& v_{i, 1}(k)=w_{i}^{u}(k-1) u(k)
\end{aligned}
$$

where $\mathrm{j}=1, \ldots, \mathrm{n}$, and $\mathrm{i}=1,2 \ldots, \mathrm{n}$. Let $\mathrm{l}=1,2, \ldots, \mathrm{n}+1$.

The output of a hidden unit at the hidden layer is:

$$
h_{i, l}\left(v_{i, l}(k)\right)=\left(1-\left(\frac{v_{i, l}(k)-b_{i, l}}{a_{i}}\right)^{2}\right) * \operatorname{EXP}\left\langle-\left(\frac{v_{i, l}(k)-b_{i, l}}{a_{i, l}}\right)^{2}\right\rangle
$$

where $1=1, \ldots, \mathrm{n}+1$

$$
x_{i}(k)=\prod_{l=1}^{n+1} h_{i, l}\left(v_{i, l}(k)\right)
$$

The context unit output is:

$$
x_{j}^{c}(k)=a_{i, j} x_{i}(k-1)+\alpha x_{j}^{c}(k-1)
$$

The final output of the network is: 


$$
y(k)=\frac{\sum_{i=1}^{n} w_{i}^{y}(k-1) x_{i}(k)}{\sum_{i=1}^{n} x_{i}(k)}
$$

\subsection{Training of the ERWNN}

This paper derives the DBP algorithm to train the proposed self feedback ERWNN. This is our second motivation in this paper. In this case the feedback vector, $x^{c}(k)=\left\{x_{j}^{c}(k)\right\}, \quad$ is $\quad a_{i, j} x_{j}(k-1)+\alpha x_{j}^{c}(k-1)$, which is a function of $w^{x}(k-2) x(k-2)+w^{u}(k-2) u(k-2)$. Therefore, $x_{j}^{c}(k)$ depends on the weights of the previous time instants. When an input-output pair, $\left(\mathrm{u}(\mathrm{k}), \mathrm{y}_{\mathrm{d}}(\mathrm{k})\right)$, is presented to the network at time $\mathrm{k}$, the error function at the network output defined in (14) can be minimized to obtain the best solution from a set of given solutions. Accordingly, the weights are updated using DBP algorithm at each time step k as follows:

$$
E_{k}=\frac{1}{2}\left[y_{d}(k)-y(k)\right]^{2}
$$

where $\mathrm{y}_{\mathrm{d}}(\mathrm{k})$ and $\mathrm{y}(\mathrm{k})$ are the desired and the actual outputs respectively.

$$
\begin{aligned}
& \frac{-\partial E_{k}}{\partial w_{i}^{y}(k-1)}=\frac{-\partial E_{k}}{\partial y(k)} * \frac{\partial y(k)}{\partial w_{i}^{y}(k-1)}=\left(y_{d}(k)-y(k)\right) * \frac{\partial x_{i}}{\sum_{i=1}^{n} x_{i}(k)} \\
& \frac{-\partial E_{k}}{\partial w_{i}^{u}(k-1)}=\frac{-\partial E_{k}}{\partial y(k)} * \frac{\partial y(k)}{\partial x_{i}(k)} * \frac{\partial x_{i}(k)}{\partial w_{i}^{u}(k-1)} \\
& \frac{\partial y}{\partial x_{i}}=\frac{\left(\sum_{i=1}^{n} x_{i}(k)\right) * w_{l}^{y}(k-1)-\left(\sum_{i=1}^{n} w_{l}^{y} x_{i}(k)\right)}{\left(\sum_{i=1}^{n} x_{i}(k)\right)^{2}}
\end{aligned}
$$

According to the above derivatives (16) becomes:

$$
\begin{aligned}
& \frac{-\partial E_{k}}{\partial w_{i}^{u}(k-1)}=\left(y_{d}(k)-y(k)\right) * \frac{\partial y}{\partial x_{i}} * \prod_{l=1}^{n} h_{i, l}\left(v_{i, l}(k)\right) \frac{\partial h_{i, 1}\left(v_{i, l}(k)\right)}{\partial w_{i}^{u}(k-1)} \\
& \text { where, } \frac{\partial h_{i, 1}(k)}{\partial w_{i}^{u}(k-1)}=\frac{\partial h_{i, 1}\left(v_{i, l}(k)\right)}{\partial v_{i, 1}(k)} * \frac{\partial v_{i, 1}(k)}{\partial w_{i}^{u}(k-1)}=\frac{\partial h_{i, 1}\left(v_{i, l}(k)\right)}{\partial v_{i, 1}(k)} * u(k) \\
& \frac{\partial h_{i, 1}\left(v_{i, l}(k)\right)}{\partial v_{i, 1}(k)}=-2 *\left\{2-\left(\frac{v_{i, 1}(k)-b_{, i}}{a_{i, l}}\right)^{2}\right\} * \frac{v_{i, 1}(k)-b_{i, l}}{\left(a_{i, l}\right)^{2}} * \operatorname{EXP}\left(-\left(\frac{v_{i, 1}(k)-b_{i, l}(k)}{a_{i, l}(k)}\right)^{2}\right) \\
& \frac{-\partial E_{k}}{\partial \boldsymbol{w}_{i, j}^{x}(k-1)}=\frac{-\partial E_{k}}{\partial y(k)} * \frac{\partial y(k)}{\partial x_{i}(k)} * \frac{\partial x_{i}(k)}{\partial w_{i, j}^{x}(k-1)}=\left(y_{d}(k)-y(k)\right) * \frac{\partial y}{\partial x_{i}} * \frac{\partial x_{i}(k)}{\partial w_{i, j}^{x}(k-1)}
\end{aligned}
$$




$$
\begin{aligned}
\frac{\partial x_{i}(k)}{\partial w_{i, j}^{x}(k-1)}=\prod_{\substack{l=1 \\
l \neq j}}^{n+1} h_{i, l}\left(v_{i, l}(k)\right) * \frac{\partial h_{i, j}\left(v_{i, j}(k)\right)}{\partial w_{i, j}^{x}(k-1)} & =\prod_{\substack{l=1 \\
l \neq j}}^{n+1} h_{i, l}\left(v_{i, l}(k)\right) * \frac{\partial h_{i, j}\left(v_{i, j}(k)\right)}{\partial v_{i, j}(k)} * \frac{\partial v_{i, j}(k)}{\partial w_{i, j}^{x}(k-1)} \\
& =\prod_{\substack{l=1 \\
l \neq j}}^{n+1} h_{i, l}\left(v_{i, l}(k)\right) * \frac{\partial h_{i, j}\left(v_{i, j}(k)\right)}{\partial v_{i, j}(k)} * x_{j}^{c}(k)
\end{aligned}
$$

The variable $x_{j}^{c}(k)$ depends on the weights of the previous time instants, $\mathrm{w}(\mathrm{k}-2)$. Accordingly, substituting (20) into (12), results (See the appendix ):

$$
\frac{\partial x_{i}(k)}{\partial w_{i, j}^{x}(k-1)}=\prod_{l=1}^{n+1} h_{i, l}\left(v_{i, l}(k)\right) * \frac{\partial h_{i, j}\left(v_{i, j}(k)\right)}{\partial v_{i, j}(k)} * a_{i, j}(k) * x_{i}(k-1)+\alpha * \frac{\partial x_{i}(k-1)}{\partial w_{i, j}^{x}(k-2)}
$$

and $\frac{\partial h_{i, j}\left(v_{i, j}(k)\right)}{\partial v_{i, j}(k)}$ can be computed as (18).

For the readers who are interested, they will discovered that the term $\alpha * \frac{\partial x_{i}(k-1)}{\partial w_{i, j}^{x}(k-2)}$ in (20) is equivalent to $\prod_{l=1}^{n+1} h_{i, l}\left(v_{i, l}(k)\right) * \frac{\partial h_{i, j}\left(v_{i, j}(k)\right)}{\partial v_{i, j}(k)} * w_{i, l}^{x}(k-1) * \frac{\partial x_{i}(k-1)}{\partial w_{i, j}^{x}(k-2)}$ using the DBP with $x_{j}^{c}(k)=a_{i, j} x_{j}(k-1), \quad \alpha=0$. Although these two terms do not provide exactly the same search, the former can provide an infinite impulse response. The derivative of (14) to the translation parameter of a wavelet function, b, and to the dilation parameter of a wavelet function, a, can be derived as follows:

$$
\begin{gathered}
\frac{-\partial E_{k}}{\partial b_{i, g}(k)}=\frac{-\partial E_{k}}{\partial y(k)} * \frac{\partial y(k)}{\partial x_{i}(k)} * \frac{\partial x_{i}(k)}{\partial b_{i, j}(k)} \\
=\left(y_{d}(k)-y(k)\right) * \frac{\partial y}{\partial x_{i}} * \sum_{g=1}^{n+1}\left(\prod_{\substack{j=1 \\
j \neq g}}^{n+1} h_{i, j}\left(v_{i, j}(k)\right) * \frac{\partial h_{i, g}\left(v_{i, g}(k)\right)}{\partial b_{i, g}(k)}\right) \\
\frac{\partial h_{i, g}(k)}{\partial b_{i, g}(k)}=2 *\left\{2-\left(\frac{v_{i, g}(k)-b_{i, g}}{a_{i, g}}\right)^{2}\right\} *\left(\frac{\left(v_{i, g}(k)-b_{i, g}\right)}{\left(a_{i, g}\right)^{2}}\right) * E X P\left(-\left(\frac{v_{i, g}(k)-b_{i, g}}{a_{i, g}}\right)^{2}\right) \\
\frac{-\partial E_{k}}{\partial a_{i, g}(k)}=\frac{-\partial E_{k}}{\partial y(k)} * \frac{\partial y(k)}{\partial x_{i}(k)} * \frac{\partial x_{i}(k)}{\partial a_{i, g}(k)}=\left(y_{d}(k)-y(k)\right) * \frac{\partial y(k)}{\partial x_{i}(k)} * \sum_{g=1}^{n+1}\left(\prod_{\substack{j=1 \\
j \neq g}}^{n+1} h_{i, j}(k) * \frac{\partial h_{i, g}(k)}{\partial a_{i, g}(k)}\right)
\end{gathered}
$$

where,

$$
\frac{\partial h_{i, g}(k)}{\partial a_{i, g}(k)}=2 *\left(2-\left(\frac{v_{i, g}(k)-b_{i, g}}{a_{i, g}}\right)^{2}\right) *\left(\frac{\left(v_{i, g}(k)-b_{i, g}\right)^{2}}{\left(a_{i, g}\right)^{3}}\right) * \operatorname{EXP}\left(-\left(\frac{v_{i, g}(k)-b_{i, g}}{a_{i, g}}\right)^{2}, \quad g=n+1 .\right.
$$

for $(g=1,2, \ldots, n)$ : 


$$
\begin{aligned}
& \frac{\partial h_{i, g}(k)}{\partial a_{i, g}(k)}=-2 *\left(\frac{\left(v_{i, g}(k)-b_{i, g}\right)}{a_{i, g}^{3}}\right) *\left\{\left(a_{i, g} \partial v_{i, g}(k) / \partial a_{i, g}\right)-\left(v_{i, g}(k)-b_{i, g}\right)\right\} *\left\{2-\left(\frac{v_{i, g}(k)-b_{i, g}}{a_{i, g}}\right)^{2}\right\} * \\
&\left(\frac{\left(v_{i, g}(k)-b_{i, g}\right)^{2}}{\left(a_{i, g}\right)^{3}}\right) * \operatorname{EXP}\left(-\left(\frac{v_{i, g}(k)-b_{i, g}}{a_{i, g}}\right)^{2}\right.
\end{aligned}
$$

where, $\frac{\partial v_{i, g}(k)}{\partial a_{i, g}(k)}=w_{i, g}^{x}(k-1) * x_{j}(k-1)$.

\section{SIMULATION RESULTS IN MODELING DYNAMIC SYSTEMS}

Simulation experiments using the proposed ERWNN were conducted on modeling dynamic systems. Since the ERWNN is employed to perform this simulation, only control signal is fed as input to the network. The developed DBP described above is used to adapt the parameters of the ERWNN using the differences between the desired and the actual outputs e(k). The RMS error as defined in (25) was computed for the trained network using the test data.

$$
R M S \text { error }=\sqrt{\frac{1}{T} \sum_{k=1}^{T}(Y d[k]-y[k])^{2}}
$$

where $Y d[k]$ and $Y[k]$ respectively, are the desired and the actual outputs and $T$ is the number of samples during the test period.

For comparison reasons, the number of hidden/context units, which should be at least be equal the order of the system to be modeled, was taken as six to enable the conventional ERNN and the proposed ERWNN to model and control most practical systems. The training times for the DBP-trained ERNN and the DBP-trained proposed ERWNN were the same in the following simulation examples. Both the two networks are tested using two types of processes, one is linear and the other is nonlinear.

\subsection{Linear Systems Modeling}

Although the proposed ERWNN with the DBP can model complex systems, it has first been used to identify a linear system to test the soundness of the proposed network. The third-order linear system employed in this simulation has one real pole and two complex poles [13]:

$$
G(S)=\frac{\omega}{\left(S+t_{1}\right)\left[\left(S+t_{2}\right)^{2}+\omega^{2}\right]}
$$

Its discrete form is:

$$
y(k)=A_{1} y(k-1)+A_{2} y(k-2)+A_{3} y(k-3)+B_{1} u(k-1)+B_{2} u(k-2)+B_{3} u(k-3)
$$

With sampling period $\mathrm{T}=0.08$ Sec. and the parameters $\mathrm{t}_{2}=1.0, \mathrm{t}_{1}=2.5$ and $\omega=2 \pi / 2.5$, the coefficients of (27) are $\mathrm{A}_{1}=2.627771, \mathrm{~A}_{2}=-2.333261, \mathrm{~A}_{3}=0.697676, \mathrm{~B}_{1}=0.017203$ and $\mathrm{B}_{2}=-0.030862, \mathrm{~B}_{3}=0.014086$. The training normalized squares set of 600 data points, was produced randomly to the system model with zero initial conditions and 
recording the output data. After training the two networks, they were tested using a different random set of 100 points and the responses of the conventional ERNN and the proposed ERWNN are obtained and the RMS errors were computed using the RMS error defined in (25). They are $4.04 * 10^{-3}$ and $1.38 * 10^{-3}$ respectively.

\subsection{Non-linear Systems Identifications}

The ERNN and the proposed ERWNN were also tested using a non-linear dynamic system. The same structure used for the above linear system modeling was employed. The nonlinear system model is [27] and [28]:

$$
\begin{array}{r}
y(k)=\left(0.8-0.5 \exp \left(-y^{2}(k-1)\right)\right) y(k-1)- \\
\left(0.3+0.9 \exp \left(-y^{2}(k-1)\right)\right) y(k-2)+ \\
0.1 \sin (3.1415926(k-1))+e(k)
\end{array}
$$

where e $(\mathrm{k})$ was a Gaussian white noise sequence with zero mean and variance equal to 0.01 . A data base of 600 data points was created using (28) (initial condition: $y(0)=y(k-$ $1)=0.1$ ). The first 500 data were employed as training data. The last 100 data were reserved as new data test for the trained model. Both the ERNN and the proposed ERWNN were trained using noise $\mathrm{e}(\mathrm{k})$ as the input and $\mathrm{y}(\mathrm{k})$ as the desired output. The DBP was employed as the training algorithm. The response of the trained two networks to the new data are depicted in Fig. 2 and Fig. 3 and the RMS error defined in (25) was computed for the testing data of the ERNN and the proposed ERWNN and was found 0.76 and 0.53 respectively.

Using the conventional ERNN and the proposed ERWNN with DBP algorithm, modeling simulation RMS errors for the above experiments are listed in Table I. The table shows that best results have been obtained using the proposed ERWNN network. It also depicts that using the two networks to obtain similar RMS error values, e. g. $1.38 * 10^{-3}$ and 0.53 respectively, the number of hidden/context units of the conventional ERNN should be increased.

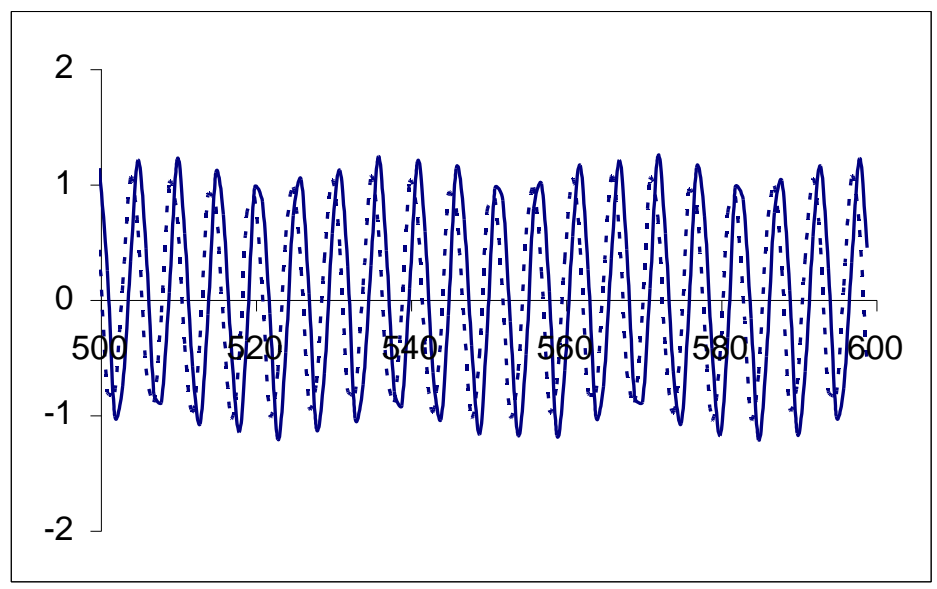

Fig. 2. Response of the ERNN (the non-linear system defined in (28)). 


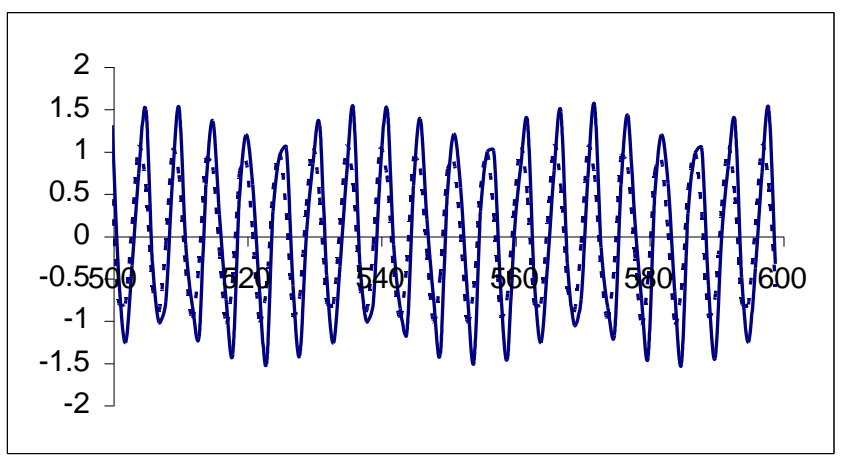

Fig. 3. Response of the proposed ERWNN (the non-linear system defined in (28)).

Table 1: Modelling simulation results.

\begin{tabular}{|l|c|c|c|c|}
\hline \multicolumn{1}{|c|}{ Cases } & ERNN & ERWNN & \multicolumn{2}{|c|}{ hidden/context units } \\
\cline { 4 - 5 } & & & ERNN & ERWNN \\
\hline The $3^{\text {nd }}$-order linear system & $4.04 * 10^{-3}$ & $1.38^{*} 10^{-3}$ & 9 & 6 \\
\hline The nonlinear system & 0.76 & 0.53 & 11 & 6 \\
\hline
\end{tabular}

\subsection{Identifiability, Generality and Stability Analysis}

Identifiability, generality, and stability are very important features for a neural network to be a universal approximator and to be suitable for control purposes. These features can be defined as follows. First, identifiability basically consists of two parts, parameter convergence and parameter consistency. The former usually does not pose a serious problem, because it depends on the convergence of the optimization technique used. The latter, on the other hand, requires that a unique set of parameter values results from the identification method, which can be difficult to admire. Second, generality is measured in terms of the Mean Squared Error (MSE) over a test or validation set of data not previously seen by the network [29]. A small MSE means good generalization ability. If this error increases after training has progressed for some time, over-fitting or over-training is said to happen [30]. Based on our simulation results depicted above, the RMS errors obtained are very small values and each error value dose not increases after training phase. Third, stability and convergence mean that feeding a sustained new input pattern to a network should not delete previously learned information [31]. The stability of the ERNN proven in [17] and [32] can be applied to the proposed ERWNN with ease as follows. The recurrent networks [10], [11], [33], and [34] are given in a static model [32] defined as follows:

$$
\tau \frac{d v_{i}}{d t}=-v_{i}+f_{i}\left(\sum_{j=1}^{n} w_{i j} v_{j}+u_{i}\right)_{, \mathrm{i}=1,2, \ldots, \mathrm{n}}
$$

where $\tau_{\text {is }}$ a positive parameter, $v_{i}$ is the state of neuron $i$ with $n e t_{i}=\sum_{j=1}^{n} w_{i j} v_{j}+\theta_{i}$ being its local field state, $f_{i}($.$) the activation function of neuron$ 
$\mathrm{i}, u_{i}$ the external input, $w_{i j}$ the link weight between the neuron $\mathrm{i}$ and neuron $\mathrm{j}$ respectively. Recurrent networks usually employs sigmoid functions or (in our proposal) wavelet functions defined in (6). The former employs the parameter $\lambda$ for controlling the steepness of the sigmoid curve while the latter uses the dilation parameter for controlling the width of the wavelet function to capture the low and high frequencies of the process be controlled. Equation (29) was generalized as [32]:

$$
\tau \frac{d v}{d t}=-v+F(W v+u)
$$

where $v=\left(v_{1}, v_{2}, \ldots, v_{n}\right)$ is the neural network state vector, $W=\left(w_{i j}\right)_{n x n}$ is the weight matrix and $F: R^{N} \rightarrow R^{N}$ is the nonlinear mapping associated with the network's activation functions. System (30) has at most a finite number of equilibrium states if one of the five conditions introduced by [23] is fulfilled, however, a recurrent network to be a stable network, it should fulfill the necessary condition; $R(F)=[-1,1]^{n}$.

It is clear that the globally convergent dynamics of recurrent networks has been a prerequisite for their application [33]. Because of its difficulty, there has been lack of a systematic analysis on such dynamical property [34]. The paper proposes the following test to check the stability and convergence of the proposed ERWNN. In this modeling task two sequences of 500 and 300 points respectively, one forming a sinusoidal signal and the other a superposition of two sinusoidal signals, were applied to the test process defined in (28) and the corresponding outputs recorded. This provided a training data file of 800 points. The input was normalised in the range [-1, 1]. The input signal employed to generate the training points for the proposed ERWNN network is defined as:

$$
\begin{array}{r}
\mathrm{u}(\mathrm{k})=\sin \left(\frac{2 \pi \mathrm{k}}{250}\right), 0 \leq \mathrm{k}<500, \quad \mathrm{u}(\mathrm{k})=\left(0.8 * \sin \left(\frac{2 \pi \mathrm{k}}{250}\right)\right)^{2}+ \\
0.2 * \sin \left(\frac{2 \pi \mathrm{k}}{25}\right), \quad 500 \leq \mathrm{k} \leq 800
\end{array}
$$

The sequences defined in (31) were employed to test the generality and stability/convergence of the proposed network. The output of the network and the process are obtained and the RMS error as defined in (25) was computed for the trained networks using the test data and it was 0.42 . In this test, the new input pattern (the second part of (31) to the ERWNN network did not delete previously learned information (the first part of (31)). This our third motivation in this paper.

\section{MODELING AND CONTROLLING TIME INTENSIVE DYNAMIC SYSTEMS USING THE ERWNN}

This paper uses time intensive respirator process to test the proposed control scheme. Accordingly, this section describes the model of the process, and the proposed modeling and controlling structures using the ERWNN. 


\subsection{Plant Model}

The model relating $\mathrm{S}_{\mathrm{a}} \mathrm{O}_{2}$ to $\mathrm{F}_{\mathrm{I}} \mathrm{O}_{2}$ is based on that describing the response of $\mathrm{P}_{\mathrm{a}} \mathrm{O}_{2}$ to an increment of $\mathrm{F}_{\mathrm{I}} \mathrm{O}_{2}$ [21]. The transfer function of the change of the $\mathrm{P}_{\mathrm{a}} \mathrm{O}_{2}$ to the change of the $\mathrm{F}_{\mathrm{I}} \mathrm{O}_{2}$ is described as:

$$
\frac{\Delta P_{a} O_{2}(s)}{\Delta F_{1} O_{2}(s)}=\frac{G_{p} e^{-T_{d} s}}{1+\tau_{p} s}
$$

where, $\Delta \mathrm{P}_{\mathrm{a}} \mathrm{O}_{2}$ is the change in arterial $\mathrm{O}_{2}$ partial pressure (torr), $\Delta \mathrm{F}_{\mathrm{I}} \mathrm{O}_{2}$ is the change in inspired oxygen fraction (percentage $\mathrm{O}_{2}$ ), $\mathrm{G}_{\mathrm{p}}$ is the sensitivity of $\mathrm{P}_{\mathrm{a}} \mathrm{O}_{2}$ to $\mathrm{F}_{\mathrm{I}} \mathrm{O}_{2}$ (torr /percent $\mathrm{O}_{2}$ ), $\mathrm{T}_{\mathrm{d}}$ is the system transport lag time (sec.), and $\tau_{\mathrm{p}}$ is the plant time constant (sec.).

Actual values for the parameters in (32) can be dynamically changed with time. The anticipated ranges for each parameter are: $\mathrm{G}_{\mathrm{P}}=55-550$ (Torr / percent $\left.\mathrm{O}_{2}\right), \tau_{\mathrm{p}}=30-$ $120 \mathrm{sec}$ (nominal $=60 \mathrm{sec}$.), and $\mathrm{T}_{\mathrm{d}}=15-45 \mathrm{sec}$ (nominal $=20 \mathrm{sec}$. $)$.

The actual value of $\mathrm{P}_{\mathrm{a}} \mathrm{O}_{2}$ can be computed by adding initial tension $\left(\mathrm{P}_{\mathrm{a}} \mathrm{O}_{2}(0)\right)$ to the differential partial pressure:

$$
\mathrm{P}_{\mathrm{a}} \mathrm{O}_{2}(\mathrm{~s})=\mathrm{P}_{\mathrm{a}} \mathrm{O}_{2}(0)+\Delta \mathrm{P}_{\mathrm{a}} \mathrm{O}_{2}(\mathrm{~s})
$$

The relationship between $\mathrm{P}_{\mathrm{a}} \mathrm{O}_{2}$ and $\mathrm{S}_{\mathrm{a}} \mathrm{O}_{2}$ can be described by the sigmoid $\mathrm{O}_{2}$ dissociation curve [35]. The $\mathrm{O}_{2}$ dissociation curve can be displaced by a number of physiological factors, i.e., $\mathrm{P}_{\mathrm{a}} \mathrm{CO}_{2}, \mathrm{pH}$, temperature, and 2,3 diphosphoglycerate levels. The model assumes that the chemical reaction of $\mathrm{O}_{2}$ binding to blood is standard, i.e., $\mathrm{P}_{\mathrm{a}} \mathrm{CO}_{2}=40$ torr, $\mathrm{pH}=7.4$, and temperature is $37^{\circ} \mathrm{C}$. Hence, $\mathrm{S}_{\mathrm{a}} \mathrm{O}_{2}$ can be computed from $\mathrm{P}_{\mathrm{a}} \mathrm{O}_{2}$ using the standard $\mathrm{O}_{2}$ dissociation curve. Severinghaus [36] provides a simple two-constant equation for the standard $\mathrm{O}_{2}$ dissociation curve valid for $\mathrm{S}_{\mathrm{a}} \mathrm{O}_{2}>$ 0.3 with a maximum error of 0.55 percent at 0.9877 saturation;

$$
\mathrm{S}_{\mathrm{a}} \mathrm{O}_{2}=\frac{1}{2340 \oint_{\left.P_{a} O_{2}{ }^{3}+150 P_{a} O_{2}\right]^{-1}+1}}
$$

The simulation diagram of the system is shown in Fig. 4. The anticipated ranges of the model parameters are given in [21]:

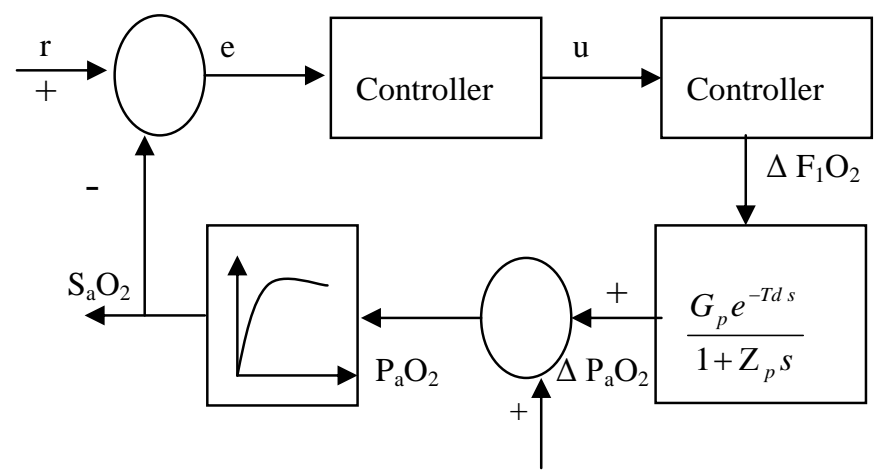

Fig. 4. Respiratory system diagram. 


\subsection{ERWNN-Based Indirect Control Scheme}

The model reference control strategy is an indirect adaptation method [37], [38], and [39]. This method is characterized by online identification to form a process model, which is then used to perform controller design. This has the advantage of separating the model identification and controller design stages for easier analysis. A combination of available model identification and controller design techniques can then be used to produce a controller with the desired characteristics [40], and [41].

The proposed control scheme in this paper, ERWNN-ICS, is illustrated in Fig. 5. The control scheme must perform two major tasks: (1) process identification and (2) process control. The former is achieved by using the proposed ERWNN as an identifier (ERWNNI) to estimate the dynamics of the controlled process. The latter is achieved by using the proposed ERWNN as a controller (ERWNNC) to generate the control signals. This results a new version of indirect control scheme. This our fourth motivation in this paper.

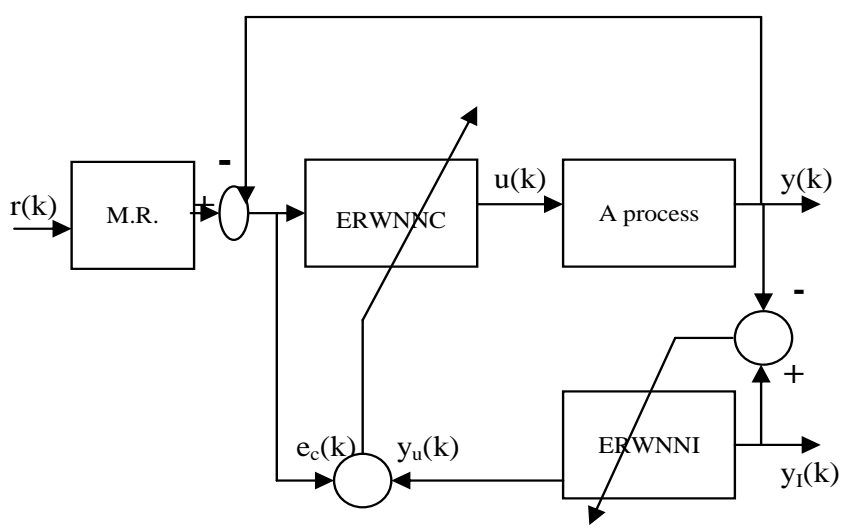

Fig. 5. The proposed indirect control architecture using ERWNN.

The reference model (RM) specifies the desired performance of the control system. The controller is designed such that the actual output of the system will track the desired output of the reference model. To produce a desired output that will be compared with the actual output, we employed the following reference trajectory that takes the form of a smooth first-order transition from the initial actual value of the output $\left(\mathrm{S}_{\mathrm{a}} \mathrm{O}_{2}\right)$ towards the known reference [38]. That is:

$$
\mathrm{y}_{\mathrm{r}}(\mathrm{k})=\alpha \mathrm{y}_{\mathrm{r}}(\mathrm{k}-1)+(1-\alpha) \mathrm{r}(\mathrm{k})
$$

where, $r(k)$ is the reference value (the set point) and $\alpha \in[0,1]$ is a constant. The closer $\alpha$ is to 0 the faster the transition. However, $\mathrm{r}(\mathrm{k})$ can be directly applied due to the slow response of the respiratory system.

\section{TRAINING THE ERWNNI AND ERWNNC}

Briefly, our goal is to minimize the error function, $\mathrm{E}_{\mathrm{I}}$. For a training pattern, $\mathrm{E}_{\mathrm{I}}$ is proportional to the sum of the squares of the difference between the actual system output $\mathrm{S}_{\mathrm{a}} \mathrm{O}_{2}$. Suppose $\mathrm{E}_{\mathrm{I}}$ is: 


$$
E_{I}=\frac{1}{2}\left[y_{p}(k)-y_{I}(k)\right]^{2}
$$

$y_{p}$ is the process output $S_{a} O_{2}$ and $y_{I}$ is the output of the ERWNNI. Then the gradient of error defined in (36) with respect to any weight in the identifier network $\mathrm{w}_{\mathrm{I}}$ becomes:

$$
\frac{\partial E_{I}}{\partial w_{I}}=e_{I}(k) \frac{\partial e_{I}(k)}{\partial w_{I}}=-e_{I}(k) \frac{\partial y_{I}(k)}{\partial w_{I}}
$$

where $\mathrm{e}_{\mathrm{I}}(\mathrm{k})=\mathrm{y}_{\mathrm{p}}(\mathrm{k})-\mathrm{y}_{\mathrm{I}}(\mathrm{k})$ is the error between the system and the ERWNNI outputs. The parameters can be adjusted using the following rule:

$$
w_{I}(k+1)=w_{I}(k)+\Delta w_{I}(k)=w_{I}(k)+\eta_{I}\left(-\frac{\partial E_{I}}{\partial w_{I}}\right)
$$

where $\eta_{I} \in[0,1]$ is a learning rate.

Similarly, the parameters of the controller can be adapted as described above. Although the system is identified, the sensitivity term $\mathrm{y}_{\mathrm{u}}(\mathrm{k})$ is unknown. This paper also derives the sensitivity value to design the parameters of the controller ERWNNC using the plant model ERWNNI. This is our fifth motivation in this paper. Once the trained model of the process is obtained, we assume the sensitivity can be approximated as:

$$
\mathrm{y}_{\mathrm{u}}(\mathrm{k})=\frac{\partial y_{p}(k)}{\partial u(k)} \approx \frac{\partial y_{I}(k)}{\partial u(k)}
$$

The plant sensitivity can be derived as follows:

$$
\frac{\partial y(k)}{\partial u(k)}=\frac{\left[\sum_{i=1}^{n} \frac{\partial w_{i}^{y}(k-1) x_{i}(k)}{\partial u(k)}\right]-y(k) \cdot \sum_{i=1}^{n} \frac{\partial x_{i}(k)}{\partial u(k)}}{\sum_{i=1}^{n} x_{i}(k)}=\frac{\left[\sum_{i=1}^{n} \partial w_{i}^{y}(k-1) \frac{\partial x_{i}(k)}{\partial u(k)}\right]-y(k) * \sum_{i=1}^{n} \frac{\partial x_{i}(k)}{\partial u(k)}}{\sum_{i=1}^{n} x_{i}(k)}
$$

where, $\frac{\partial x_{i}(k)}{\partial u(k)}=\prod_{l=1}^{n} h_{i, l}\left(v_{i, l}(k)\right) * \frac{\partial h_{i, n+1}\left(v_{i, n+1}(k)\right)}{\partial u(k)}$

$$
\frac{\partial h_{i, n+1}\left(v_{i, n+1}(k)\right)}{\partial u(k)}=\frac{\partial h_{i, n+1}(k)}{\partial v_{i, n+1}(k)} * \frac{\partial v_{i, n+1}(k)}{\partial u(k)}=\frac{\partial h_{i, n+1}\left(v_{i, n+1}(k)\right)}{\partial v_{i, n+1}(k)} * w_{i}^{u}(k-1)
$$

$\frac{\partial h_{i, n+1}\left(v_{i, n+1}(k)\right)}{\partial v_{i, n+1}(k)}$ can be computed similar to (18).

\section{TESTING}

Simulation experiments using the proposed ERWNN were conducted on two test schemes, one is a process modeling and the other is a process control. The RMS error defined in (25) was computed for the trained network using the test data.

\subsection{The Process Modeling Simulation Results}

The proposed ERWNN with one input unit, a six of hidden/context units and one output unit was used to identify the $\mathrm{O}_{2}$ uptake system. Accordingly, the network has 
48 weights to be updated with the DBP. Simulation was performed using the following cases:

Case $(1): T_{s}=15 \mathrm{sec}, \tau_{p}=60 \mathrm{sec}, \mathrm{T}_{\mathrm{d}}=20 \mathrm{sec}$ and $\mathrm{G}_{\mathrm{p}}=123$ torr $/$ percent $\mathrm{O}_{2}$.

Case(2): $\mathrm{T}_{\mathrm{s}}=15 \mathrm{sec}, \tau_{\mathrm{p}}=60 \mathrm{sec}, \mathrm{T}_{\mathrm{d}}=20 \mathrm{sec}$ and $\mathrm{G}_{\mathrm{p}}=250 \mathrm{torr} /$ percent $\mathrm{O}_{2}$.

Case(3): $T_{s}=15 \mathrm{sec}, \tau_{p}=60 \mathrm{sec}, \mathrm{T}_{\mathrm{d}}=20 \mathrm{sec}$ and $\mathrm{G}_{\mathrm{p}}=300$ torr $/$ percent $\mathrm{O}_{2}$.

Ten thousands square pulses (step inputs) were applied to the $\mathrm{O}_{2}$ uptake system and the corresponding outputs recorded. This provided a training data file of 10000 points. The inputs were normalised in the range $0-1$. The amplitudes and the frequencies of the inputs were changed pseudo-randomly after 60 time steps. The trained model was tested using data that had not been employed for training. The test signal and the actual output of the process are obtained using the ERNN and ERWNN and the RMS error defined in (25) was computed and its values were $2.21 \times 10^{-3}$ and $0.9 \times 10^{-3}$ respectively.

\subsection{Indirect Control Using ERWNN Simulation Results}

The indirect control architecture using ERWNN explained in sections IV, ERWNNICS, was tested over a range of plant parameters. The testing is performed at two values of plant gain $\left(\mathrm{G}_{\mathrm{p}}=123,250\right.$ torr/ percent $\left.\mathrm{O}_{2}\right)$. The first value of $\mathrm{G}_{\mathrm{p}}$ is tested with dynamic combinations of plant time constant $\left(\tau_{\mathrm{p}}=30,60,90 \mathrm{sec}\right)$, and transport lag $\left(\mathrm{T}_{\mathrm{d}}=15,20,25 \mathrm{sec}\right)$, and the second value of $\mathrm{G}_{\mathrm{p}}$ is tested with fixed combination of plant time constant and transport lag $\left(\tau_{\mathrm{p}}=60 \mathrm{sec}, \mathrm{T}_{\mathrm{d}}=20 \mathrm{sec}\right)$, but the plant gain was halved (at $\mathrm{t}=10 \mathrm{~min}$.) in the presence of a disturbance to test the regulation capabilities of the proposed controller. The values of the plant time constant and transport lag were chosen such as, they provide the greatest degree of mismatch between the plant and the model.

In general, noise signals can be classified into impulse noise, thermal noise, shut noise, and additive white Gaussian noise. Measurements were corrupted with additive white Gaussian noise generated using the algorithm written in the appendix [35]. The output of the controller network is limited to give an $\mathrm{F}_{\mathrm{I}} \mathrm{O}_{2}$ between $21 \%$ and $100 \% \mathrm{O}_{2}$. The controller was commanded to raise $\mathrm{S}_{\mathrm{a}} \mathrm{O}_{2}$ from an initial value of about 0.8 to a reference value of 0.95 and to maintain at the new setpoint. The reference trajectory, chooses $\alpha=0.68$. The sampling period of the results is $15 \mathrm{sec}$.

Two network with one input unit, six hidden/context units and 48 weight were employed in this test. One stand for the identifier and the other performs the controller.

\section{1- Simulation results with $\mathrm{G}_{\mathrm{p}}=123$ torr / percent $\mathrm{O}_{2}$}

With gain $\left(\mathrm{G}_{\mathrm{p}}=123\right.$ torr / percent $\left.\mathrm{O}_{2}\right)$ three combination of the time constant and the time delay are tested as follows:

case (1): $\tau_{\mathrm{p}}=60 \mathrm{sec}$ and $\mathrm{T}_{\mathrm{d}}=20 \mathrm{sec}$.

case (2): $\tau_{\mathrm{p}}=30 \mathrm{sec}$ and $\mathrm{T}_{\mathrm{d}}=15 \mathrm{sec}$.

case (3): $\tau_{\mathrm{p}}=90 \mathrm{sec}$ and $\mathrm{T}_{\mathrm{d}}=25 \mathrm{sec}$.

The RMS errors were calculated using (25) and their values are $6.28 * 10^{-3}, 6.75^{*} 10^{-3}$, and $1.14 * 10^{-2}$, for the three cases.

\section{2- Simulation results at disturbances presence}

Testing the regulation capabilities of the proposed ERWNN-ICS in the presence of a disturbance such that the plant gain was halved at ( $\mathrm{t}=10 \mathrm{~min}$.) from 250 to 125 torr / percent $\mathrm{O}_{2}$, indicates the soundness of the proposed scheme. The RMS error was 
computed using (25) and its value is $7.99 * 10^{-3}$. The outputs and the control signal are shown in Fig. 6, which shows that the error was eliminated within 0.85 min. That assures the soundness of the proposed indirect control scheme using the ERWNN. For comparison reasons, this experiment was performed using the conventional ERNN_ICS. The outputs and the control signal are shown in Fig. 7, which shows that the error was eliminated within $1.25 \mathrm{~min}$ and the RMS error obtained was $12.00 * 10^{-3}$. In sense of recovering time and the RMS error defined in (25), Table II shows the results obtained using the proposed indirect control scheme and that obtained in [21], and [42].

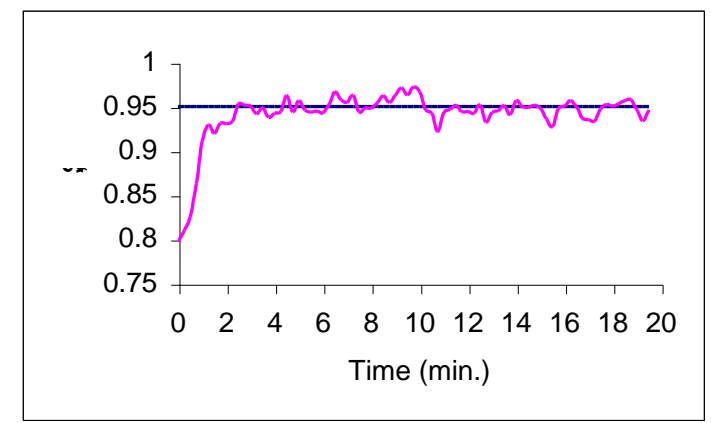

Fig. 6-a. The process output.

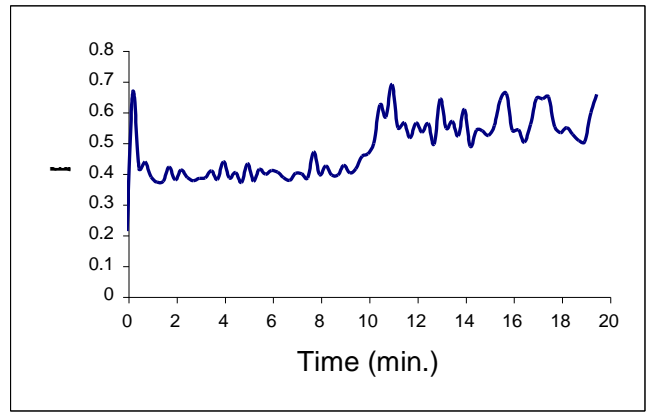

Fig. 6-b. The control signal.

Fig. 6. The process output and the control signal using the ERWNN-ICS.

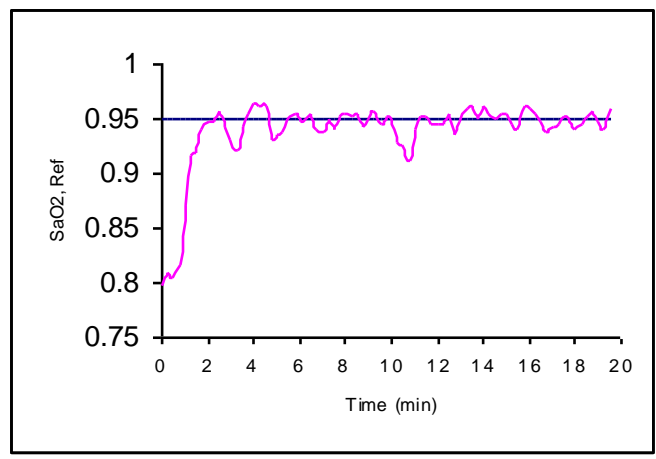

Fig. 7-a. The process output. 


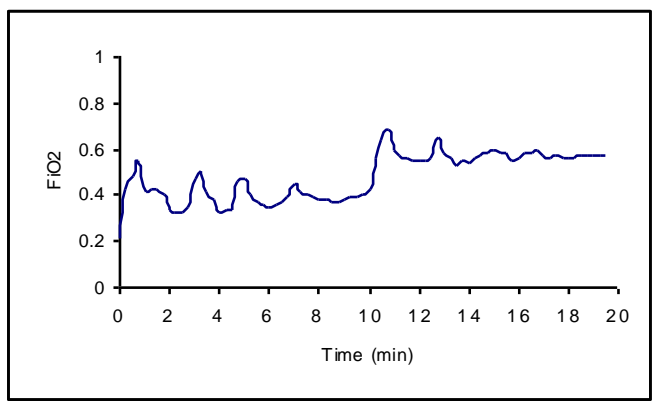

Fig. 7-b. The control signal.

Fig. 7. The process output and the control signal using the ERNN-ICS.

Table 2: Comparison with results published in [21] and [42].

\begin{tabular}{|l|c|c|}
\hline Controller type & Time required (min.) & $\begin{array}{c}\text { Disturbance rejection } \\
\text { (RMS) errors }\end{array}$ \\
\hline PI & At least 10.00 & $20.50^{*} 10^{-3}$ \\
\hline MMAC & 3.00 & $11.00^{*} 10^{-3}$ \\
\hline FNN-ICS & 2.5 & $8.59 * 10^{-3}$ \\
\hline ERNN-ICS & 1.25 & $12.00^{*} 10^{-3}$ \\
\hline ERWNN-ICS & 0.85 & $7.99 * 10^{-3}$ \\
\hline
\end{tabular}

As a final conclusion, Table 2 depicts that best results have been achieved using the ERWNN-ICS compared with the conventional PI-controller, MMAC controller [21], the FNN-ICS and the ERNN-ICS, [42] for controlling the $\mathrm{O}_{2}$ uptake system. In the sense of RMS errors and time required to reject the disturbances shown in the table, the ERWN-ICS is promising scheme for controlling real time intensive processes in noisy conditions and disturbances. The application areas for the introduced technology are very wide, however, the author can conclude the major application of this technology as follows:

- A universal tool for function approximation

- Real time learning of unknown functions

- Modelling and control time intensive real time processes

- Data compression

- Financial and economic analysis

- Biomedical engineering

\section{CONCOLUSIONS}

This paper introduced a new version of Elman networks named ERWNN. Compared with the traditional ERNN, the proposed ERWNN has three notable features, local accuracy, generalization capability, stability and convergence property. The former two features are inherited from replacing the global property of sigmoid functions 
used at the hidden layer of ERNN by the multiresolution property of wavelets while the latter is gained from employing few context/hidden units, which memorizes the previous actions of the hidden units and can be considered to function one-step time delay. This paper developed a model reference control scheme that belongs to indirect adaptive control. The scheme consists of two ERWNNs, one performs the controller and the other stands for the system's model. This paper also derived the plant sensitivity and DBP algorithm. The former is employed for adjusting the parameters of the developed controller and the latter is used to train both the two networks of the proposed indirect control scheme, ERWNN-ICS. Structurally, the number of hidden/context units (should at least be equal to the order of the system be modeled ) of an ERWNN employed was fixed to be six to control the actual processes with ease. The control of the oxygen delivery to mechanically ventilated hypoxic patients is best achieved using the proposed indirect control scheme, ERWNN-ICS, compared with the conventional PI-controller, MMAC-controller, FNN-ICS, and ERNN-ICS.

\section{REFERENCES}

[1] K. S. Narendra and K. Parthasarathy, "Identification and control of dynamical systems using neural networks," IEEE Transactions on Neural Networks, vol. 1, pp. 4-27, 1990.

[2] K. J. Hunt, D. Sbarbaro, R. Zbikowski and P. J. Gawthrop, "Neural networks for control systems-A survey," Automatica, vol. 28, pp. 1083-1112, 1992.

[3] G. G. Lendaris and T. T. Shannon, "Application considerations for the DHP methodology," Proceedings of International Joint Conference on Neural Networks-98, (IJCNN-98), Anchorage, IEEE press, pp. 1013-1018, 1998.

[4] H. A. Awad, Fuzzy neural networks for modeling and controlling dynamic systems. PhD. Thesis, Intelligent Systems Laboratory, School of Engineering, Cardiff University, Cardiff, UK, 2001.

[5] D. E. Rumelhart and J. L., McClelland. Parallel distributed processing: Explorations in the micro-structure of cognition, vol. 1, Cambridge, Mass: MIT Press, 1986.

[6] J. L. Elman, "Finding structure in time," Cognitive Science, vol. 14, pp. 179-211, 1990.

[7] T. Kohonen, .Self-organised and associative memory. Springer Verlag: Berlin, 1989.

[8] G. A. Carpenter, S. Grossberg and D. B. Rosen, "Fuzzy ART: Fast stable learning and categorization of analogue patterns by an adaptive resonance system," Neural Networks, vol. 4, pp. 759-771, 1991.

[9] G. G. Lendaris and C. Paintz, "Training strategies for critic and action neural networks in Dual Heuristic programming method," Proceedings of International Joint Conference on Neural Networks-97, (IJCNN-97), Houston, pp. 712-717, 1997.

[10] D. T. Pham and X. Liu, "Dynamic systems modeling using recurrent neural networks," Journal of Systems Eng., vol. 2, pp. 90-97, 1992.

[11] J. Hertz, A. Krogh and R. G. Palmer, Recurrent neural networks: Introduction to the theory of neural computations. California: Addison-Wesley, chapter 7, 1991. 
[12] C.-M. Kuan, Estimation of neural models. Ph.D. Thesis, University of California, San Diego, 1989.

[13] D. T. Pham and H. X. Liu, "Training of Elman networks and dynamic system modeling," International Journal of syst. Sci., vol. 27, no., 2, pp. 221-226, 1996.

[14] N. M. El-Rabaie, H. A. Awad and T. A. Mahmoud, "Wavelet fuzzy neural network-based predictive control system, "IEEE MELECON, Dubrovnik, Croatia, pp. 307-310, 2004.

[15] D. W. C. Ho, P.-A. Zhang and J. Xu, "Fuzzy wavelet networks for function learning," IEEE Transactions on Fuzzy Systems, vol. 9, pp. 200-211, 2001.

[16] C.-H. Lee and C.-C. Teng, "Identification and control of dynamic systems using recurrent fuzzy neural networks," IEEE Transactions on Fuzzy Systems, vol. 8, pp. 349-366, 2000.

[17] H. Wersing, W. J. Beyn and H. Ritter, "Dynamical stability conditions for recurrent neural networks with understanding piecewise linear transfer functions," Neural Computing, vol. 13, pp. 1811-1825, 2001.

[18] Y. Mitamura, T. Mikami, H. Sugawara and C. Yoshimoto, "An optimally controlled respirator," IEEE Trans. on Biomed Eng., vol. BME-18, pp. 330-337, 1971.

[19] Y. Sun, I. Kohane and A. Stark, "Fuzzy logic assisted control of inspired oxygen in ventilated newborn infants," Proc. Annu. Symp. Comput. Appl. Med. Care, pp. 756-761, 1994.

[20] Y. Sun, I. Kohane and A., Stark, "Computer-assisted adjustment of inspired oxygen concentration improves control of oxygen saturation in newborn infants requiring mechanical ventilation," Journal of pediatrics, vol. 131, pp. 754-756, 1997.

[21] C. Yu and I. He, "Improvement in arterial oxygen control using multiple-model adaptive control procedures," IEEE Trans. Biomed Eng., vol. BME-34, p. 567, 1986.

[22] N. M. El-Rabaie, H. A. Awad, and K. T. Abo-Alam, "Arterial oxygen control using the enhanced fuzzy neural scheme," Al Azher University Engineerin Journal, AUEJ, Cairo, Egypt, 2003.

[23] I. Daubechies, "Wavelet transform, time-frequency localization and signal analysis," IEEE Transactions on Information Theory, vol 36, pp. 961-1005, 1990.

[24] Q. Zhang, "Using wavelet networks in nonparametric estimation," IEEE Transactions on Neural networks, vol. 8, pp. 277-236, 1997.

[25] O. Yacine and D. Gerard, "Initialization by selection for wavelet training," Neural Computing, vol. 34, pp. 131-143, 2000.

[26] K. C. Kan and K. W. Wong, "Self-constriction algorithm for synthesis of wavelet networks," Electronic Letter, vol. 34, pp. 1953-1955, 1998.

[27] S. Chen and S. a. Billings. Neural networks for modeling and identification: Advances in intelligent control, Edited by C. J. Harris, London, pp. 101-102, 1994.

[28] D. T. Pham and X. Liu, "Identification of linear and nonlinear dynamic systems using recurrent neural networks," Artificial Intelligence in Engineering, vol. 8, pp. 67-75, 1993.

[29] S. McLoone and G. Irwin, "Non-linear optimization of RBF networks," International Journal of Systems Science, vol. 29, pp. 179-189, 1998. 
[30] D. R. Hush and B. G. Horne, "Progress in supervised neural networks," IEEE Signal Processing Magazine, pp. 8-39, 1993.

[31] G.A. Carpenter and S. Grossberg, "ART2: Self-organization of stable category recognition codes for analogue input patterns," Applied Optics, vol. 26, pp. 49194930, 1987.

[32] H. Qiao, J. P. Jigen Z.-B. Xu and B. Zhang, "A reference model approach to stability analysis of neural networks," IEEE Transactions on Systems, MAN, and Cybernetics-PARTB: Cybernetics, vol. 33, pp. 925-936, 2003.

[33] F. J. Pineda, "Generalization of back-propagation to recurrent neural networks," Phys. Rev. Lett., vol. 59, pp. 2229-2232, 1987.

[34] R. Rohwer and B. Forrest, "Training time-dependence in neural networks,"In Proc. $1^{\text {st }}$ IEEE Int. Conference, Neural Networks, vol. 2, San Diego, CA, pp. 701708, 1987.

[35] C. Yu, An arterial oxygen saturation controller, Master's thesis, , Rensselaer Polytech. Inst., Tory, NY, 1986.

[36] J. W. Severinghaus, "Simple, accurate equations for human blood $\mathrm{O}_{2}$ dissociation computation," J. Appl. Phys. : Resp. Environ. Exercise Physiol., vol. 46, pp. 559602, 1979.

[37] C.G. Moore, Indirect adaptive fuzzy controllers. PhD. Thesis, Dept. of Aeronautics and Astronautics, University of South Hampton, UK, 1991.

[38] K. Astrom and B. Wittenmark, Adaptive control. Reading, MA: Addison-Wesley Pub. Co., 1989.

[39] C.-F. Juang and C.-T. Lin "A recurrent self-organizing neural fuzzy inference network," Proceedings of the sixth IEEE International Conference on Fuzzy Systems, vol. III, Barcelona, Spain, 1997.

[40] A. Tsakonas and G. Dounias, "Intelligent applications- Hybrid computational intelligence schemes in complex domains: An extended review," In Lecture Notes in Computer Science 2002, vol. 2308, pp. 494-512, Springer, 2002.

[41] Y.-C. Chen and C.-C. Teng, "A model reference control structure using a fuzzy neural network," Fuzzy sets and Systems, vol. 73, pp. 291-312, 1995.

[42] K. T. Abo Alam, Fuzzy neural control for hypoxemia. Faculty of Electronic Eng., Menoufia Unv., Egypt, 2005.

\section{THE APPENDIX}

In (20) assume that, $\phi=\prod_{\substack{l=1 \\ l \neq j}}^{n+1} h_{i, l}\left(v_{i, l}(k)\right) * \frac{\partial h_{i, j}\left(v_{i, j}(k)\right)}{\partial v_{i, j}(k)}$. That equation becomes, $\frac{\partial x_{i}(k)}{\partial w_{i, j}^{x}(k-1)}=\phi x_{j}^{c}(k)$. Further, $x_{j}^{c}(k)=\frac{1}{\phi} \frac{\partial x_{i}(k)}{\partial w_{i, j}^{x}(k-1)}$. At time instant (k-1), It becomes: $x_{j}^{c}(k-1)=\frac{1}{\phi} \frac{\partial x_{i}(k-1)}{\partial w_{i, j}^{x}(k-2)}$. Substituting this equation into (12), results:

$\frac{1}{\phi} \frac{\partial x_{i}(k)}{\partial w_{i, j}^{x}(k-1)}=a_{i, j} x_{j}(k-1)+\alpha \frac{1}{\phi} \frac{\partial x_{i}(k-1)}{\partial w_{i, j}^{x}(k-2)}$

Multiplying the resulted equation by $\phi$, results: 
$\frac{\partial x_{i}(k)}{\partial w_{i, j}^{x}(k-1)}=\prod_{l=1}^{n+1} h_{i, l}\left(v_{i, l}(k)\right) * \frac{\partial h_{i, j}\left(v_{i, j}(k)\right)}{\partial v_{i, j}(k)} * a_{i, j}(k) * x_{i}(k-1)+\alpha * \frac{\partial x_{i}(k-1)}{\partial w_{i, j}^{x}(k-2)}$.

the

resulted equation is equivalent to (21).

The responses of the oxygen uptake system are corrupted with additive white Gaussian noise generated using the following algorithm [35]:

$\mathrm{SN}=\mathrm{RAN}$

$\mathrm{SN}=2.4 *(\mathrm{SN}-0.5)$

$\mathrm{SAO} 2=\mathrm{SAO} 2+\mathrm{SN} / 100$

\section{نوع جديد من شبكات المان للنمذجة والتحكم في النظم الايناميكية}

تعتبر شبكة ELMAN من الثبكات العصبية ذات التخذية الراجعة والتي تستخدم بشكل اساسي في

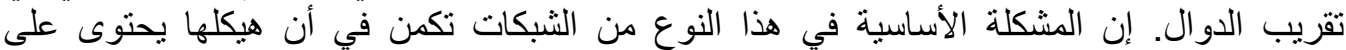

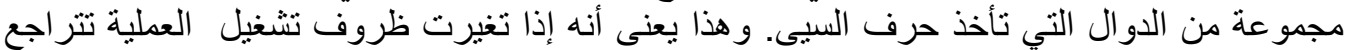

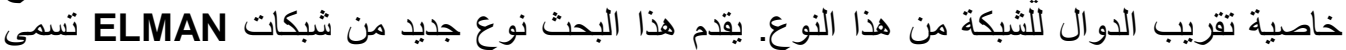
ERWNN

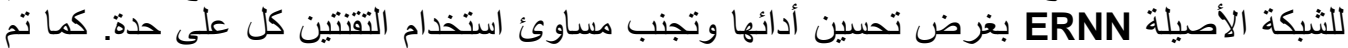

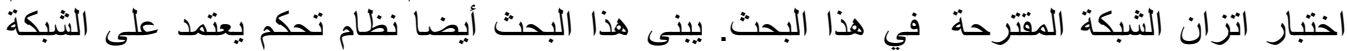

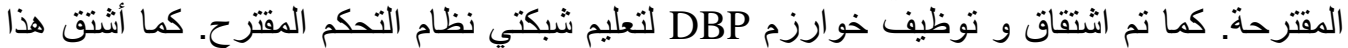
البحث The plant sensitivity و التي يعتبر من أهم المتغير ات لضبط لفيط عناصر الحاكم المستخدم في

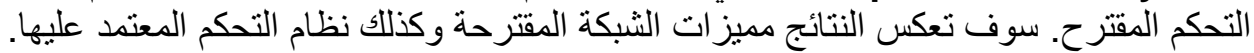

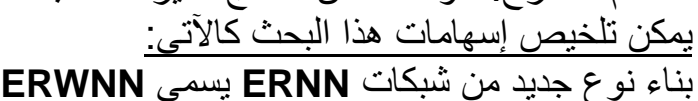
اشتقاق خوارزم DBP لتعليم الثبكة المقترحة

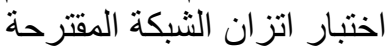
بناء نظام تحكم جديد معتمدا على الثبكة المقترحة يسمى ERWNN-ICS ومقارنة أدائه بمجمو عة من

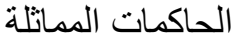
اثتنقاق The plant sensitivity والتي يعتبر من أهم المتغيرات لضبط عناصر الحاكم المستخدم

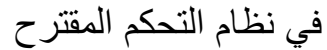

Dr. Hamdy A. Awad was born in Egypt, 1964. He received the B.Sc. degree in Industrial Electronics, and the M. Sc. degree in Adaptive Control Systems from Faculty of Electronic

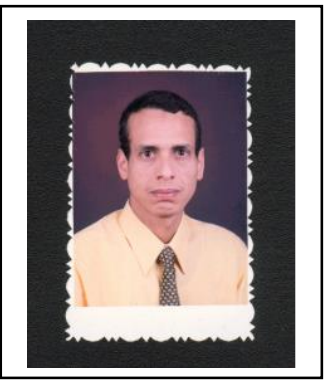
Engineering, Menouf, Menoufia University, Egypt in 1988 and 1994 respectively. He received the Ph.D. degree in Artificial Intelligent Systems in 2001 from School of Engineering, Cardiff University, Cardiff-Wales, England. Since July 2001.

He has been with the Faculty of Electronic Engineering, Menoufia University, where he is currently a lecturer of Control Engineering in Industrial Electronics Engineering and Control Dept.

Dr. Awad interests in intelligent control systems, machine learning, and their applications in control systems, fault diagnosis and medical engineering. 